\title{
Mixed Key Management Using Hamming Distance for Mobile Ad-Hoc Networks
}

\author{
Seok-Lae Lee ${ }^{1}$, In-Kyung Jeun ${ }^{1}$, and Joo-Seok Song ${ }^{2}$ \\ ${ }^{1}$ KISA, 78 Garak-Dong, Songpa-Gu, Seoul, 138-803, Korea \\ \{sllee, ikjeun\}@kisa.or.kr \\ ${ }^{2}$ Yonsei University, 134 Shinchon-Dong, Seodaemoon-Gu, Seoul, 120-749, Korea \\ jssong@emerald.yonsei.ac.kr
}

\begin{abstract}
Unlike fixed networks, mobile ad-hoc networks have diverse characteristics, such as dynamic topologies, bandwidth-constrained links, energy-constrained operation, limited physical security, etc. Due to these characteristics their security requirements are different from those of fixed networks. This paper presents a method of authenticating the nodes in the ad-hoc network and securely transmitting information in consideration of the characteristics of the mobile ad hoc network. To this end, this paper proposes a method of combining asymmetric and symmetric cryptography to enhance the efficiency of secret key management for authentication and secure channel establishment. In particular, this paper proposes a method that introduces the concept of Hamming Distance to share the secret keys between the nodes. According to this proposal, secure communication between the nodes is possible when one node manages only $\log _{2} N$ secret keys.
\end{abstract}

Keywords: Hamming Distance, Certificate, Public-key, Ad-hoc Network, Secret Key.

\section{Introduction}

Mobile ad-hoc networks provide convenient infra-free communications over wireless channels. Recently, mobile ad-hoc wireless networking technology has been applied to various applications such as military tactical networks, sensor networks, and disaster area networks [5]. But it is difficult to implement mobile ad-hoc networks due to physical constraints such as the limited power range and small memory of mobile device, and security issues [6] such as authentication. Recently, there are many efforts to resolve the constraints of mobile ad-hoc networks with various security issues [7, 8], [9, [1].

In [7, 8], 9], Luo et al distributed the functions of certification authority through a threshold secret sharing method [3, 4] and scalable multi-signature mechanism. In [11, Capkun et al proposed a fully self-organized public-key management system that doesn't need any trusted authorities in mobile ad-hoc network. The authors presented methods by which the authentication mechanism of the mobile ad-hoc network can act on its own without the help of the centralized 
TTP(Trusted Third Party), but since it acts on the basis of the public-key algorithm, it needs to be improved in terms of actual implementation or efficiency in consideration of the performance of the nodes.

Accordingly, this paper proposes a method of ensuring the security and reliability of the mobile ad-hoc network by providing authentication and secure communication based on symmetric cryptography while acting independently from the centralized TTP. Besides, the concept of Hamming Distance [1] is introduced to show that it is possible to securely transmit information between the nodes, though one node only manages $\log _{2} N$ secret keys. In this paper, $N$ means the number of nodes in the network.

This paper is organized as follows: In section 2, we explain the concepts of Hamming Distance. In section 3, we present a secure communication channel establishing method. In section 4, we propose the key management method. In section 5 and 6 , we propose a path construction algorithm and analyze its performance.

\section{Concepts}

First, we explain the basic characteristics of Hamming Distance between two integers and the concept of Hamming Distance for improving the number of secret keys exchanged between one node and others as $\log _{2} N$. We use the following two definitions to simplify our explanation.

(1) The $I D$ s of two nodes, $a$ and $b$, are defined as $a_{I D}\left(\in Z_{N}\right)$ and $b_{I D}\left(\in Z_{N}\right)$ and Hamming Distance between these $I D$ s are defined as $H D(a, b)$ (or $H D$ ).

(2) A node $a$ stores and manages the secret keys exchanged with other nodes. At this time, node $a$ from the viewpoint of other nodes is defined as parent node $(P N)$ and the other nodes is defined as child nodes $(C N)$. Besides, one of $C N$ is defined as $C N_{i}$. Each node meets the following condition : $H D\left(P N, C N_{i}\right)=1$.

Theorem 1. For $a \in Z_{N}$, define $R_{a}=\left\{x \in Z_{N} \mid H D(a, x)=1\right\}$, then $\left|R_{a}\right|=\log _{2} N$ where $Z_{n}=\{0,1, \ldots, N-1\}, N=2^{m}, m>0$

Proof. For an integer $a \in Z_{N}$, the number of integers which Hamming Distance in comparison with a is "1" is the same as the number of integers which Hamming weight in comparison with " 0 " is "1". Generally, the number of integers that Hamming weight in comparison with " 0 " is $r$ is computed to ${ }_{m} C_{r}$. Here, $m$ means $\log _{2} N$ and $C$ means combination. Thus, for a number a, the number of integers that Hamming Distance is "1" is equivalent to $m\left(=\log _{2} N\right)$ as ${ }_{m} C_{1}$.

In this paper, each node is assigned an element over $Z_{N}$ as the unique $I D$ of its public-key certificate. At this time, this $I D$ is different concept with the serial number of public-key certificate x.509 v3 10 and is managed by using the extension field within certificate. To construct a secure communication path and manage a secret key efficiently, each node is assigned its own $I D$ by the 


\begin{tabular}{|c|l|l|l|l|l|l|l|l|}
\hline Node ID & 000 & 001 & 010 & 011 & 100 & 101 & 110 & 111 \\
\hline 000 & & $\bullet$ & $\bullet$ & & $\bullet$ & & \multicolumn{2}{|c|}{$R_{001}$} \\
\hline 001 & $\bullet$ & & & $\bullet$ & & $\bullet$ & 5 & \\
\hline 010 & $\bullet$ & & & $\bullet$ & & & $\bullet$ & \\
\hline 011 & & $\bullet$ & $\bullet$ & & & & & $\bullet$ \\
\hline 100 & $\bullet$ & & & & & $\bullet$ & $\bullet$ & \\
\hline 101 & & $\bullet$ & & & $\bullet$ & & & $\bullet$ \\
\hline 110 & & & $\bullet$ & & $\bullet$ & & & $\bullet$ \\
\hline 111 & & & & $\bullet$ & & $\bullet$ & $\bullet$ & \\
\hline
\end{tabular}

Fig. 1. The set of $I D$ s with $H D=1$

administrator of the ad-hoc network. This $I D$ has particular inter-relationship with some nodes by using $H D$. As for this matter, we explain in section 5 and 6 through a path construction algorithm and simulation. In addition, the number of nodes that a node has to manage will become $\log _{2} N$.

Figure 1 briefly describes the characteristics explained in the above. Figure 1 represents the relationship between integers with $H D=1$ about the integer set of $N=8$. Namely, the number of integers that Hamming Distance with "001" is " 1 " is $3\left(=\log _{2} 8\right)$ such as $\{" 000 ", " 011 ", " 101 "\}$. At this time, if $I D$ of $P N$ is "001", $I D$ s of $C N$ are $\{" 000 ", " 011 ", " 101 "\}$. Namely, $R_{001}$ becomes $\{" 000 ", " 011 "$, "101"\}. According to Theorem 1, a PN only stores and manages the secret keys belonging to $\log _{2} N$ nodes.

\section{Secure Communication Channel}

This section uses the concept of Hamming Distance to describe how to establish a secure channel for securely transmitting information between nodes in the adhoc network. For simplicity's sake, it is assumed that $N$ is 8 , and information is transmitted through the secure channel between two nodes $\left(N_{000}\right.$ and $\left.N_{110}\right)$.

For starters, each node identifies the nodes with $H D=1$. In figure 2, nodes with $H D=1$ in comparison with $N_{000}$ are $\left\{N_{001}, N_{010}, N_{100}\right\}$. To establish a secure channel between nodes, each node exchanges secret keys with those nodes with $H D=1$. To ensure security at this time, each node uses the public-key of the other node to encrypt and transmit its secret key. In other words, nodes exchanging secret keys with node $N_{000}$ are $\left\{N_{001}, N_{010}, N_{100}\right\}$, and the secret keys are $\left\{K_{(000,001)}, K_{(000,010)}, K_{(000,100)}\right\}$. At this time, $K_{(000,001)}$ and $K_{(001,000)}$ is the same secret key. And node $N_{000}$ may exchange a different secret key with each node to enhance security. In this method, all nodes exchange the secret keys with nodes that satisfy $H D=1$. On the assumption that secret keys were exchanged like this, the method of establishing a secure channel for securely transmitting information between two nodes will be explained. That is, a communication path between $S n$ and $D n$ in figure 2 is determined to securely 


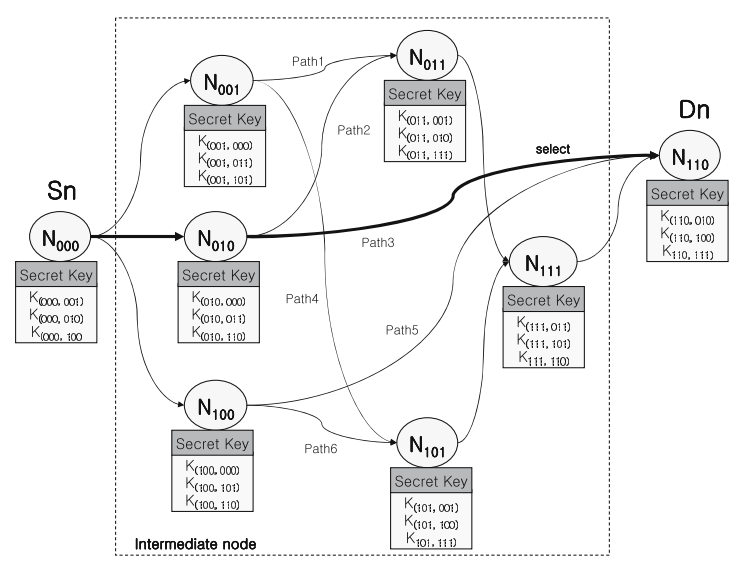

Fig. 2. The concept of a communication path construction

transmit information from $N_{000}$ to $N_{110}$. There are six paths in figure 2 and one of these paths is selected to transmit information.

\section{Secure Key Management}

A node that wants to participate in the ad-hoc network needs to cross certify and exchange the encryption key for secure communication with other nodes. This section proposes a method that combines asymmetric and symmetric cryptography as the method of managing secret keys necessary for cross certification and encryption in this ad-hoc network. Asymmetric cryptography is used when the public-key certificate for an ad-hoc network administrator is issued from the TTP, and when an initial key is installed on each node. The initial key is used when a node participating in the ad-hoc network exchanges secret keys with other nodes. Symmetric cryptography is used for authentication and secure communication between nodes.

In general, in symmetric cryptography, as all nodes must share secret keys with each other, the number of secret keys managed by one node must be $(N-1)$ like figure 3(a). However, in our paper, we show that it is possible to get the secure communication even if only $\log _{2} N$ secret keys are exchanged like figure 3(b). This paper divides the key management method into four stages: administrator's preparation, node initialization under the administrator's control, secret key exchange, and secure communication.

\subsection{Preparation}

In the preparation stage the administrator of an ad-hoc network registers in the centralized TTP to initialize nodes. In other words, the administrator generates a public-key pair using his own PC, stores the private-key in his tamper-proofing device, sends the public-key to TTP, and gets the certificate issued from TTP. 


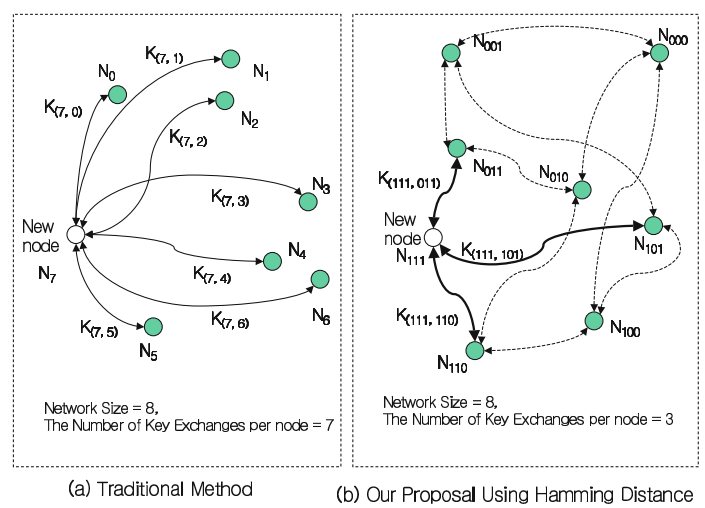

Fig. 3. Traditional method vs. Hammming Distance method

\subsection{Node Initialization}

For a node to participate in an ad-hoc network, it must be identified to the existing network participants. To this end, the node must have the information necessary for identifying and confirming itself to the nodes already participating in the ad-hoc network (authentication information). In the node initialization state this authentication information is given to the nodes. Node initialization is performed by the administrator.

Node initialization is as follows. (1) The administrator connects to TTP. TTP verifies the administrator's public-key certificate(administrator authentication). (2) A secure channel is established for secure communication between the TTP and administrator (secure channel establishment). (3) The administrator generates the public-key pair corresponding to the node, stores the private-key in the tamper-proofing area of the node and sends the public-key to the TTP (node's public-key pair generation). (4) The TTP generates a certificate to the public-key of the node, and then sends its own certificate and node's certificate together to the node (node's certificate issuance). At this time, the TTP's certificate is used to authenticate other nodes while participating in the ad-hoc network, and the node's certificate is used to authenticate itself for other nodes. In particular, the node's certificate in this stage complies with x, 509 v3 certificate profile, but includes diverse information, such as $I D$, IP address, device usage, and device validity date, to restrict the use of the node's certificate.

\subsection{Secret Key Exchange}

In this stage, the secret keys for secure communication between nodes are shared. In general, for secure communication between nodes, all nodes must own secret keys necessary for data encryption. In sharing these secret keys, it is important to minimize the impact of the leakage of the secret keys in the network to an intruder and provide end-to-end security. As the best method, if the network size is $N$, one node needs to exchange secret keys with $(N-1)$ other nodes, and 


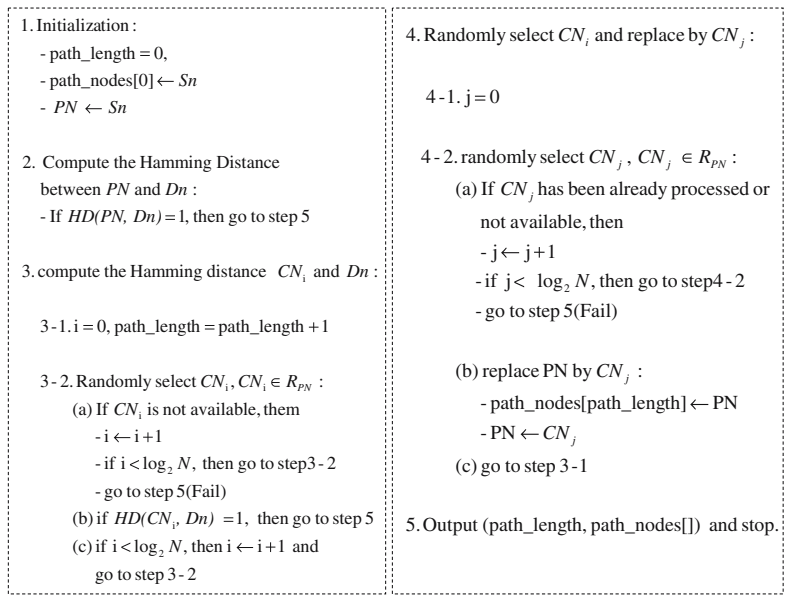

Fig. 4. The path construction algorithm

bears the burden of managing $(N-1)$ secret keys. Accordingly, there must be some trade-off among the network security, the burden of exchanging secret keys between nodes, and the quantity of secret keys managed by one node.

As the concept of Hamming Distance is introduced, it seems possible to securely transmit information in the ad-hoc network only if secret keys are shared between those nodes with $H D=1$. Figure 3(b) illustrates how secret keys are shared between nodes. Since one node only needs to manage $\log _{2} N$ secret keys in the above method, the burden of the node will be reduced to a considerable extent.

\subsection{Secure Communication}

For secure communication between two nodes, a communication path between the two nodes must first be established. Section 5 describes how to establish the path. Once the path is established, the secret keys owned by the nodes on respective paths are used for communication between two nodes. That is, secure communication between two nodes can be explained by means of figure 2 . The assumption in figure 2 is that the communication path from $N_{000}$ to $N_{110}$ is $\left\{N_{000} \rightarrow N_{010} \rightarrow N_{110}\right\}$. First of all, as $N_{000}$ and $N_{010}$ share the secret key $K_{(001,010)}, N_{000}$ uses the secret key $K_{(000,010)}$ to transmit information to $N_{010}$, whereas $N_{010}$ uses a secret key $K_{(010,110)}$ to transmit information to $N_{110}$.

\section{Path Construction Algorithm Using Hamming Distance}

A path construction algorithm refers to find one path establishing the secure communication channel from $S n$ (Source node) to $D n$ (Destination node). In figure 2, there are six paths from $S n$ to $D n$ and only One of these paths is constructed as the secure communication path from $S n$ to $D n$. 
We present an algorithm illustrated in figure 4 to search a path from $S n$ to $D n$ by using the characteristics of Hamming Distance. First, the terminology that used in figure 4 will be explained briefly. Path_nodes represents the path information from $S n$ to $D n$ in the process of constructing a path. The algorithm proposed in this paper is divided into two parts. One is computing Hamming Distance between $D n$ and the $C N_{i}$ of $P N$. The other is a process to replace $P N$ with one node among $C N$ of $P N$ in case that $H D \neq 1$. If $H D(P N, D n)=1$ or $H D\left(C N_{i}, D n\right)=1$, the path construction algorithm is successfully terminated. If $H D \neq 1, P N$ is replaced by randomly choosing one of $C N$. And then by using $C N$ of the replaced $P N$, it also verifies to satisfy $H D=1$. As repeating this computation, a path can be found.

In general, the path construction algorithm is similar to routing algorithm in mobile ad-hoc network. In this paper, algorithm performance is defined in the perspective of how to accurately find a path. By using the algorithm performance evaluation method proposed in [7, it is defined as the following.

$$
\begin{aligned}
P_{b} & \left(T_{H D}, r, Z_{N}\right) \\
& =\frac{\left|\left\{(S n, D n) \in Z_{N} \times Z_{N}: S n \rightarrow_{s u c} D n\right\}\right|}{\left|\left\{(S n, D n) \in Z_{N} \times Z_{N}: S n \rightarrow_{\text {tri }} D n\right\}\right|}
\end{aligned}
$$

Here, $T_{H D}:$ A path construction algorithm using $H D, \quad r:\left(N-N_{r}\right) / N$

$S n \rightarrow$ tri $D n$ : Path searching trials, $S n \rightarrow_{\text {suc }} D n:$ Path constructing cases

\section{Simulation Results for the Path Construction Algorithm}

The main goal of this simulation is to verify a completion level of our proposed algorithm in figure 4 . Thus, this simulation intends to analyze the success probability of path construction in case that the number of nodes on mobile ad-hoc network is smaller than $N\left(=2^{m}\right)$. Namely, we analyze the success possibility by evaluating the performance of the proposed algorithm according to

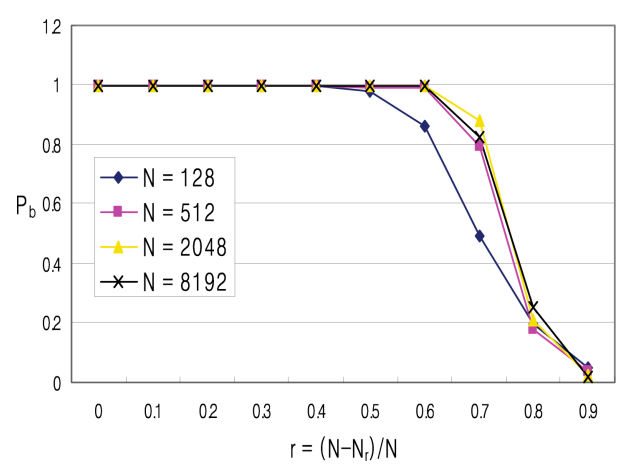

Fig. 5. Success probability $\left(P_{b}\right)$ vs. Node reducing factor $(r)$ 
the node reducing rate $r$. Figure 5 represents the success probability of constructing a path versus node reducing factor $r(=\{0.1, \ldots, 0.9\})$ according to $N$ $(=\{128,512,2048,8192\})$. As seen in figure 5 , a path is constructed about $97.6 \%$ success probability at confidence interval [2] $99 \%$ in case of $r=0.5$. If $r<0.5$, the success probability will be high. As a result, the performance of our algorithm is outstanding.

\section{Conclusion}

We used asymmetric and symmetric cryptography to propose a method of secure communication between nodes over mobile ad-hoc network. Besides, the method based on Hamming Distance was proposed as the method of exchanging keys between nodes so that one node manages only $\log _{2} N$ secret keys. The performance of our method was evaluated through simulation. According to the result of the simulation, the probability of this algorithm successfully finding a path was above $97.9 \%$ at $99 \%$ confidence interval, in case that $r$ is smaller than 0.5. In addition, our method can minimize the impact of the leakage of some secret keys on the entire ad-hoc network as individual nodes own different secret keys. However, Our method doesn't guarantee the end-to-end security directly between source and destination nodes in this paper. Accordingly, additional research needs to be made into this area.

\section{References}

1. R. Hamming: Coding and Information Theory, Prentice-Hall, 1980.

2. Raj Jain: The art of computer systems performance analysis, John Wiley \& Sons, Inc., 1991.

3. A. Herzberg, S. Jarecki, H. Krawczyk, M. Yung: Proactive Secret Sharing, or: how to cope with perpetual leakage, Advances in Cryptography - Crypto 95' Proceedings, LNCS Vol 963, 1995.

4. Y. Frankel, P. Gemmell, P.-D. MacKenzie, and M. Yung: Optimal-Resilience Proactive Public-Key Cryptosystems, IEEE Symp. on Foundations of Computer Science, 1997.

5. K. Fokine: Key Management in Ad Hoc Networks, LiTH-ISY-EX-3322-2002, 2002.

6. F. Stajano and R. Anderson: The Resurrecting Duckling : Security Issues for Adhoc Wireless Networks, Proc. seventh Int'l workshop security protocols, 1999

7. H. Luo and S. Lu: Ubiquitous and Robust Authentication Services for Ad Hoc Wireless Networks, Technical Report 200030, UCLA Computer Science Department, Oct. 2000.

8. J. Kong, P. Zerfos, H. Luo, S. Lu, and L. Zhang: Providing robust and ubiquitous security support for MANET, Proc. ninth Int'l conf. Network Protocols, Nov. 2001.

9. H.Luo, P. Zerfos, J. Kong, S. Lu, and L. Zhang: Self-securing Ad Hoc Wireless Networks, Seventh IEEE Symp. on Computers and Communications, 2002.

10. R. Housley, W. Polk, W. Ford, and D. Solo: Internet X.509 Public Key Infrastructure Certificate and CRL Profile, IETF RFC3280, April 2002.

11. S. Capkun, L.Buttyan and J.-P. Hubaux: Self-Organized Public-Key Management for Mobile Ad Hoc Networks, IEEE Trans. on mobile computing, vol. 2, No. 1, Jan./Mar. 2003. 